\title{
Symplectic Connections of Ricci Type and Star Products
}

\author{
Michel Cahen ${ }^{1}$, Simone Gutt ${ }^{12}$, and Stefan Waldmann ${ }^{3}$ \\ 1 Département de Mathématique \\ Université Libre de Bruxelles \\ Campus Plaine, C. P. 218 \\ Boulevard du Triomphe \\ B-1050 Bruxelles \\ Belgium \\ mcahen@ulb.ac.be, sgutt@ulb.ac.be \\ 2 Université Paul Verlaine - Metz \\ Département de Mathématique LMAM \\ Ile du Saulcy \\ F-57045 Metz Cedex 01 \\ France \\ gutt@poncelet.univ-metz.fr \\ 3 Fakultät für Mathematik und Physik \\ Albert-Ludwigs-Universität Freiburg \\ Physikalisches Institut \\ Hermann Herder Strasse 3 \\ D 79104 Freiburg \\ Germany \\ Stefan. Waldmann@physik . uni-freiburg. de
}

Summary. In this article we relate the construction of Ricci type symplectic connections by reduction to the construction of star product by reduction yielding rather explicit descriptions for the star product on the reduced space.

\section{Introduction}

Deformation quantization [4] is a formal deformation - in the sense of Murray Gerstenhaber [18] — of the algebraic structure of the space of smooth functions on a manifold $M$; it yields at first order in the deformation parameter a Poisson structure on $M$. When this Poisson structure is non-degenerate, i.e. when the manifold is symplectic, deformation quantization at second order yields a symplectic connection on $M$ [22].

On a symplectic manifold $(M, \omega)$, symplectic connections always exist but are not unique. The curvature $R^{\nabla}$ of such a connection $\nabla$ splits [23] under the 
action of the symplectic group, (when the dimension of $M$ is at least 4), into two irreducible components $R^{\nabla}=E^{\nabla}+W^{\nabla}$ with $E^{\nabla}$ completely determined by the Ricci tensor of the connection. A symplectic connection is said to be of Ricci type if $R^{\nabla}=E^{\nabla}$.

Marsden-Weinstein reduction, see e.g. [1, Sect. 4.3], is a method in symplectic geometry to construct a symplectic manifold $(M, \omega)$ - called the reduced space - from a bigger one $(P, \mu)$ and the extra data of a coisotropic submanifold $C$ in $P$.

Under some further asumptions, one can sometimes reduce connections $[2,3]$, i.e. define a symplectic connection $\nabla^{M}$ on $M$ from a connection $\nabla^{P}$ on $P$. In particular, any Ricci-type connection on a simply connected $2 n(\geq 4)$ dimensional manifold can be obtained [11] by reduction from a flat connection $\nabla$ on a $2 n+2$ dimensional manifold $P$, with the coisotropic codimension 1 submanifold $C$ defined by the zero set of a function $F: P \rightarrow \mathbb{R}$ whose third covariant derivative vanishes.

One way to describe the algebra of functions on the reduced space is the use of BRST methods. Jim Stasheff participated actively to the development of this point of view, see e.g. [17] among many others. Reduction of deformation quantization has been studied by various authors $[6,8,13,14,16,20]$. In particular, a quantized version of BRST methods was introduced in [8] to construct reduction in deformation quantization. We use here those methods to define a star product on any symplectic manifold endowed with a Ricci type connection.

In this context we rely both on the work of Murray and on the work of Jim and we are very happy and honoured to dedicate this to them.

In section 1, we recall some basic properties of Ricci type symplectic connections. Section 2 introduces a natural differential operator of order 2 on a symplectic manifold endowed with a symplectic connection. In section 3, we recall the expression of the Weyl-Moyal star product on a symplectic manifold with a flat connection. Section 4 explains the construction of reduced star product in our context where the reduced space $(M, \omega)$ is a symplectic manifold of dimension $2 n \geq 4$, endowed with a Ricci-type connection, and where the big space $(P, \mu)$ is of dimension $2 n+2$ with a flat connection and the related Weyl-Moyal star product. In section 5, we show some properties of the reduced star product, in particular that the connection defined by the reduced star product is the Ricci type connection.

\section{Preliminary Results on Ricci Type Connections}

In this section we recall some basic properties of Ricci type symplectic connections to explain our notation. We follow essentially [11,12] and refer for further details to the expository paper [5].

Let $(M, \omega)$ be a $2 n \geq 4$-dimensional symplectic manifold which allows for a Ricci type symplectic connection: this is a symplectic connection $\nabla^{M}$ (i.e. a linear torsion-free connection so that the symplectic 2 -form $\omega$ is parallel) 
such that the curvature tensor $R$ is entirely determined by its Ricci tensor Ric. Precisely, for $X, Y \in \Gamma^{\infty}(T M)$ we have

$$
\begin{aligned}
R(X, Y)=-\frac{1}{2 n+1} & \left(-2 \omega(X, Y) \varrho-\varrho(Y) \otimes X^{b}\right. \\
+ & \left.\varrho(X) \otimes Y^{b}-X \otimes(\varrho(Y))^{b}+Y \otimes(\varrho(X))^{b}\right),
\end{aligned}
$$

where $X^{b}=i_{X} \omega$ as usual and $\varrho$ is the Ricci endomorphism defined by

$$
\operatorname{Ric}(X, Y)=\omega(X, \varrho(Y)) .
$$

It follows that there exists a vector field $\mathrm{U} \in \Gamma^{\infty}(T M)$, a function $\mathrm{f} \in C^{\infty}(M)$ and a constant $\mathrm{K}$ such that the following identities hold

$$
\begin{gathered}
\nabla_{X}^{M} \varrho=-\frac{1}{2 n+1}\left(X \otimes \mathrm{U}^{b}+\mathrm{U} \otimes X^{b}\right), \\
\nabla_{X}^{M} \mathrm{U}=-\frac{2 n+1}{2(n+1)} \varrho^{2} X+\mathrm{f} X, \\
\operatorname{tr}\left(\varrho^{2}\right)+\frac{4(n+1)}{2 n+1} \mathrm{f}=\mathrm{K} .
\end{gathered}
$$

One of the fundamental properties of such a Ricci type connection is that $\left(M, \omega, \nabla^{M}\right)$ can be obtained from a Marsden-Weinstein reduction out of a $(2 n+2)$-dimensional symplectic manifold $(P, \mu)$ which is equipped with a flat symplectic torsion-free connection $\nabla$. In fact, we have to assume that $M$ is simply-connected; then by [11] there exists a $(2 n+1)$-dimensional manifold $\Sigma$ with a surjective submersion

$$
\pi: \Sigma \longrightarrow M
$$

together with a contact one-form $\alpha \in \Gamma^{\infty}\left(T^{*} \Sigma\right)$, i.e. $\alpha \wedge(\mathrm{d} \alpha)^{n}$ is nowhere vanishing with $\pi^{*} \omega=\mathrm{d} \alpha$, whose Reeb vector field $Z \in \Gamma^{\infty}(T \Sigma)$, defined by $\alpha(Z)=1$ and $\mathrm{i}_{Z} \mathrm{~d} \alpha=0$, has a flow such that (6) is the quotient onto the orbit space with respect to this flow. This manifold $\Sigma$ is constructed as the holonomy bundle over $M$ for a connection defined on an extension of the frame bundle. It is a $\mathbb{R}$ or $\mathbb{S}^{1}$ principal bundle over $M$ with connection one-form $\alpha$. Then we consider $P=\Sigma \times \mathbb{R}$ with $\mathrm{pr}_{1}: P \longrightarrow \Sigma$ being the canonical projection and $\iota: \Sigma \longrightarrow P$ being the embedding of $\Sigma$ as $\Sigma \times\{0\}$. The coordinate along $\mathbb{R}$ is denoted by $s$ and we set $S=\frac{\partial}{\partial s} \in \Gamma^{\infty}(T P)$. On $P$ one has the following exact symplectic form

$$
\mu=\mathrm{d}\left(\mathrm{e}^{s} \operatorname{pr}_{1}^{*} \alpha\right)=\mathrm{e}^{s} \mathrm{~d} s \wedge \operatorname{pr}_{1}^{*} \alpha+\mathrm{e}^{s} \operatorname{dpr}_{1}^{*} \alpha .
$$

Thanks to the Cartesian product structure we can lift vector fields on $\Sigma$ canonically to $P$. In particular, the lift $E$ of the Reeb vector field $Z$ (defined by $d s(E)=0$ and $\operatorname{Tpr}_{1}(E)=Z$ ) turns out to be Hamiltonian $E=-X_{H}$ with 


$$
H=\mathrm{e}^{s} \in C^{\infty}(P) .
$$

We have

$$
\mathscr{L}_{S} X_{H}=0, \quad \mathscr{L}_{S} H=H, \quad \text { and } \quad \mathscr{L}_{S} \mu=\mu,
$$

whence in particular $S$ is a conformally symplectic vector field. Moreover, $\mu\left(X_{H}, S\right)=H$ and we can rewrite $\mu$ as

$$
\mu=\mathrm{d} H \wedge \operatorname{pr}_{1}^{*} \alpha+H \operatorname{pr}^{*} \omega,
$$

where pr $=\pi \circ \operatorname{pr}_{1}: P \longrightarrow M$. Then $(M, \omega)$ is the (Marsden-Weinstein) reduced phase space of $(P, \mu)$ with respect to the Hamiltonian flow of $H$ at momentum value $H=1$, since indeed $\Sigma=H^{-1}(\{1\})$ and $\iota^{*} \mu=\pi^{*} \omega$ by (10).

Using the contact form $\alpha$ we can lift vector fields $X \in \Gamma^{\infty}(T M)$ horizontally to vector fields $\bar{X} \in \Gamma^{\infty}(T \Sigma)$ by the condition

$$
T \pi \circ \bar{X}=X \circ \pi \quad \text { and } \quad \alpha(\bar{X})=0 .
$$

Since $\mathrm{d} \alpha=\pi^{*} \omega$ we have $[\bar{X}, \bar{Y}]=\overline{[X, Y]}-\pi^{*}(\omega(X, Y)) Z$. Using also the canonical lift to $P$, we can lift $X \in \Gamma^{\infty}(T M)$ horizontally to $X^{\text {hor }} \in \Gamma^{\infty}(T P)$, now subject to the conditions

$$
T \text { pr } \circ X^{\text {hor }}=X \circ \text { pr } \quad \text { and } \quad \operatorname{pr}_{1}^{*} \alpha\left(X^{\text {hor }}\right)=0=\mathrm{d} s\left(X^{\text {hor }}\right) .
$$

We have

$$
\left[X^{\text {hor }}, Y^{\text {hor }}\right]=[X, Y]^{\text {hor }}+\operatorname{pr}^{*}(\omega(X, Y)) X_{H}
$$

as well as

$$
\left[S, X^{\text {hor }}\right]=0=\left[X_{H}, X^{\text {hor }}\right] .
$$

We shall speak of "invariance" always with respect to the flow of $X_{H}$ (or $Z$ on $\Sigma$, respectively) and of "homogeneity" always with respect to the conformally symplectic vector field $S$, e.g. a differential operator $D$ on $P$ is called homogeneous of degree $k \in \mathbb{Z}$ if $\left[\mathscr{L}_{S}, D\right]=k D$, etc.

Denote the Poisson tensor on $M$ by $\Lambda_{M}$ and the one on $P$ by $\Lambda_{P}$, respectively. We can also extend the horizontal lift to bivectors as usual. Since we have curvature, $\Lambda_{M}^{\text {hor }}$ is no longer a Poisson bivector, instead one finds

$$
\llbracket \Lambda_{M}^{\text {hor }}, \Lambda_{M}^{\text {hor }} \rrbracket=-2 \Lambda_{M}^{\text {hor }} \wedge X_{H} .
$$

From the Definition (7) of the symplectic 2 -form $\mu$ on $P$, we have the relation

$$
\Lambda_{P}=\frac{1}{H}\left(\Lambda_{M}^{\mathrm{hor}}+S \wedge X_{H}\right) .
$$

In particular, for $u, v \in C^{\infty}(M)$ we find for the Poisson brackets

$$
\left\{\operatorname{pr}^{*} u, \operatorname{pr}^{*} v\right\}_{P}=\frac{1}{H} \operatorname{pr}^{*}\{u, v\}_{M} .
$$


We are now in the position to define the flat connection $\nabla$ on $P$ by specifying it on horizontal lifts, on $S$ and on $X_{H}$. One defines [11]

$$
\begin{gathered}
\nabla_{X^{\text {hor }}} Y^{\text {hor }}=\left(\nabla_{X}^{M} Y\right)^{\text {hor }}+\frac{1}{2} \operatorname{pr}^{*}(\omega(X, Y)) X_{H}+\operatorname{pr}^{*}(t(X, Y)) S, \\
\nabla_{X^{\text {hor }}} X_{H}=\nabla_{X_{H}} X^{\text {hor }}=(\tau(X))^{\text {hor }}-\operatorname{pr}^{*}(\omega(X, \mathrm{~V})) S \\
\nabla_{X^{\text {hor }}} S=\nabla_{S} X^{\text {hor }}=\frac{1}{2} X^{\text {hor }} \\
\nabla_{X_{H}} X_{H}=\operatorname{pr}^{*} \phi S-\mathrm{V}^{\text {hor }} \\
\nabla_{X_{H}} S=\nabla_{S} X_{H}=\frac{1}{2} X_{H}, \\
\nabla_{S} S=\frac{1}{2} S
\end{gathered}
$$

where we used the abbreviations

$$
\begin{gathered}
t=\frac{1}{n+1} \text { Ric }, \\
\mathrm{V}=\frac{2}{(n+1)(2 n+1)} \mathrm{U}, \\
\phi=\frac{4}{(n+1)(2 n+1)} \mathrm{f},
\end{gathered}
$$

and $\tau$ is the endomorphism corresponding to $t$ analogously to (2). In [11], the following statement was obtained:

Theorem 1. By (18)-(23) a flat symplectic torsion-free connection $\nabla$ is defined on $P$ and $\nabla$ is invariant under $X_{H}$ and $S$. Moreover, the third symmetrized covariant derivative of $H$ vanishes.

Recall that the operator of symmetrized covariant differentiation as a derivation of the symmetric tensor product $\mathrm{D}: \Gamma^{\infty}\left(\mathrm{S}^{k} T^{*} P\right) \longrightarrow \Gamma^{\infty}\left(\mathrm{S}^{k+1} T^{*} P\right)$ is defined by

$$
(\mathrm{D} \gamma)\left(X_{1}, \ldots, X_{k+1}\right)=\sum_{\ell=1}^{k+1}\left(\nabla_{X_{\ell}} \gamma\right)\left(X_{1}, \ldots, \stackrel{\ell}{\wedge}, \ldots, X_{k+1}\right),
$$

where $X_{1}, \ldots, X_{k+1} \in \Gamma^{\infty}(T P)$ and $\gamma \in \Gamma^{\infty}\left(\mathrm{S}^{k} T^{*} P\right)$. Locally, D can be written as

$$
\mathrm{D}=\mathrm{d} x^{i} \vee \nabla \frac{\partial}{\partial x^{i}},
$$

where $\vee$ denotes the symmetrized tensor product. Then Theorem 1 means

$$
\mathrm{D}^{3} H=0 .
$$

Remark 1. The Ricci type connection on $M$ is symmetric iff $\mathrm{U}=0$ in which case $f$ turns out to be constant). This particular case has been studied in detail in [5]. 


\section{General Remarks on the Ricci Operator}

Before discussing the star products on $P$ and $M$, respectively, we introduce the following second order differential operator on a symplectic manifold with symplectic connection, which is also of independent interest. Let $(M, \omega)$ be symplectic with a torsion-free symplectic connection $\nabla$ (not necessarily of Ricci type). Then the Ricci tensor Ric $\in \Gamma^{\infty}\left(\mathrm{S}^{2} T^{*} M\right)$ can be used to define a 'Laplace'-like operator $\Delta^{\text {Ric }}$ as follows: We denote by $\operatorname{Ric}^{\sharp} \in \Gamma^{\infty}\left(\mathrm{S}^{2} T M\right)$ the symmetric bivector obtained from Ric under the musical isomorphism $\sharp$ with respect to $\omega$.

Definition 1 (Ricci operator). The Ricci operator $\Delta^{\text {Ric }}: C^{\infty}(M) \longrightarrow$ $C^{\infty}(M)$ is defined by

$$
\Delta^{\mathrm{Ric}} u=\frac{1}{2}\left\langle\operatorname{Ric}^{\sharp}, \mathrm{D}^{2} u\right\rangle,
$$

where $\langle\cdot, \cdot\rangle$ denotes the natural pairing.

If locally we write $\operatorname{Ric}^{\sharp}=\frac{1}{2} \operatorname{Ric}^{i j} \frac{\partial}{\partial x^{i}} \vee \frac{\partial}{\partial x^{j}}$ then

$$
\Delta^{\operatorname{Ric}} u=\operatorname{Ric}^{i j}\left(\frac{\partial^{2} u}{\partial x^{i} \partial x^{j}}-\Gamma_{i j}^{k} \frac{\partial u}{\partial x^{k}}\right),
$$

where $\Gamma_{i j}^{k}$ are the local Christoffel symbols of $\nabla$.

Since on a symplectic manifold we have a canonical volume form, the Liouville form $\Omega$, one can ask whether $\Delta^{\text {Ric }}$ is a symmetric operator with respect to the $L^{2}$-inner product on $C_{0}^{\infty}(M)$ induced by $\Omega$ : in general, this is not the case. However, there is an easy way to correct this. We need to recall some basic features of the global symbol calculus for differential operators on manifolds with connection, see e.g. $[9,10]$.

We denote by $(q, p)$ the canonical coordinates on $T^{*} U \subseteq T^{*} M$ induced by a local chart $(U, x)$ of $M$. Then the standard-ordered quantization of a function $f \in \mathrm{Pol}^{\bullet}\left(T^{*} M\right)$ on the cotangent bundle $\pi: T^{*} M \longrightarrow M$, which is polynomial in the momenta, is defined by

$$
\varrho_{\text {Std }}(f) u=\left.\sum_{r=0}^{\infty} \frac{1}{r !}\left(\frac{\hbar}{\mathrm{i}}\right)^{r} \frac{\partial^{r} f}{\partial p_{i_{1}} \cdots \partial p_{i_{r}}}\right|_{p=0} \mathrm{i}_{\mathrm{s}}\left(\frac{\partial}{\partial x^{i_{1}}}\right) \cdots \mathrm{i}_{\mathrm{s}}\left(\frac{\partial}{\partial x^{i_{r}}}\right) \frac{1}{r !} \mathrm{D}^{r} u,
$$

where $u \in C^{\infty}(M)$ and $\mathrm{i}_{\mathrm{s}}$ denotes the (symmetric) insertion map into the first argument. Clearly, (32) is globally well-defined and does not depend on the coordinates. The constant $\frac{\hbar}{\mathrm{i}}$ can safely be set to 1 in our context; however, we have included it for the sake of physical interpretation of $\varrho_{\text {Std }}$ as a quantization map. Then (32) gives a linear bijection $\varrho_{\text {Std }}: \operatorname{Pol} l^{\bullet}\left(T^{*} M\right) \longrightarrow \operatorname{Diffop}(M)$.

The symmetric algebra $\mathcal{S}^{\bullet}(T M)=\bigoplus_{r=0}^{\infty} \Gamma^{\infty}\left(\mathrm{S}^{r} T M\right)$ is canonically isomorphic to the polynomial functions $\operatorname{Pol}^{\bullet}\left(T^{*} M\right)$ as graded associative algebra via the "universal momentum map" $\mathcal{J}$, determined by $\mathcal{J}(u)=\pi^{*} u$ and 
$(\mathcal{J}(X))\left(\alpha_{q}\right)=\alpha_{q}\left(X_{(q)}\right)$ for $u \in \mathcal{S}^{0}(T M)=C^{\infty}(M)$ and $X \in \mathcal{S}^{1}(T M)=$ $\Gamma^{\infty}(T M)$. Thus we can rephrase (30) as

$$
\Delta^{\text {Ric }}=-\frac{2}{\hbar^{2}} \varrho_{\text {std }}\left(\mathcal{J}\left(\operatorname{Ric}^{\sharp}\right)\right),
$$

whence $\Delta^{\text {Ric }}$ is the standard-ordered quantization of the quadratic function $\mathcal{J}\left(\operatorname{Ric}^{\sharp}\right)$ on $T^{*} M$.

The standard-ordered quantization can be seen as a particular case of the $\kappa$-ordered quantization which is obtained as follows. On $C^{\infty}\left(T^{*} M\right)$ one has a Laplace operator $\Delta$ arising from the pseudo-Riemannian metric $g_{0}$, which is defined by the natural pairing of the vertical and horizontal (with respect to $\nabla)$ subspaces of $T\left(T^{*} M\right)$. Locally, $\Delta$ is given by

$$
\left.\Delta\right|_{T^{*} U}=\frac{\partial^{2}}{\partial q^{i} \partial p_{i}}+p_{k} \pi^{*}\left(\Gamma_{i j}^{k}\right) \frac{\partial^{2}}{\partial p_{i} \partial p_{j}}+\pi^{*}\left(\Gamma_{i j}^{i}\right) \frac{\partial}{\partial p_{j}} .
$$

On polynomial functions $\Delta$ is just the covariant divergence operator $[10$, Eq. (111)], i.e.

$$
\Delta \mathcal{J}(T)=\mathcal{J}\left(\operatorname{div}_{\nabla} T\right)
$$

for $T \in \mathcal{S}^{k}(T M)$ where with $\alpha_{1}, \ldots, \alpha_{k-1} \in \Gamma^{\infty}\left(T^{*} M\right)$

$$
\left(\operatorname{div}_{\nabla} T\right)\left(\alpha_{1}, \ldots, \alpha_{k-1}\right)=\operatorname{tr}\left(X \mapsto\left(\nabla_{X} T\right)\left(\cdot, \alpha_{1}, \ldots, \alpha_{k-1}\right)\right) .
$$

Locally, $\operatorname{div}_{\nabla}=\mathrm{i}_{\mathrm{s}}\left(\mathrm{d} x^{i}\right) \nabla_{\frac{\partial}{\partial x^{i}}}$. Using $\Delta$, the $\kappa$-ordered quantization is defined by $[9]$

$$
\varrho_{\kappa}(f)=\varrho_{\text {Std }}\left(\mathrm{e}^{-\mathrm{i} \kappa \hbar \Delta} f\right),
$$

where in particular the Weyl-ordered case $\varrho_{\text {Weyl }}=\varrho_{\kappa=1 / 2}$ is of interest for us. In general, we have for the formal adjoint of $\varrho_{\kappa}(f)$ with respect to $\Omega$

$$
\varrho_{\kappa}(f)^{\dagger}=\varrho_{\kappa}\left(\mathrm{e}^{-\mathrm{i} \hbar(1-2 \kappa) \Delta} \bar{f}\right)
$$

whence $\varrho_{\text {Weyl }}(f)^{\dagger}=\varrho_{\text {Weyl }}(\bar{f})$. Thus $\varrho_{\text {Weyl }}\left(\mathcal{J}\left(\right.\right.$ Ric $\left.\left.^{\sharp}\right)\right)$ gives a symmetric operator. Explicitly, one finds using (35)

$$
-\frac{2}{\hbar^{2}} \varrho_{\kappa}\left(\mathcal{J}\left(\operatorname{Ric}^{\sharp}\right)\right)=\Delta^{\mathrm{Ric}}+2 \kappa \mathscr{L}_{\operatorname{div}_{\nabla} \operatorname{Ric}^{\sharp}}+\kappa^{2} \operatorname{div}_{\nabla}^{2}\left(\operatorname{Ric}^{\sharp}\right),
$$

since no higher order terms contribute thanks to $\operatorname{Ric}^{\sharp} \in \mathcal{S}^{2}(T M)$. Moreover, $\kappa^{2} \operatorname{div}_{\nabla}^{2}\left(\operatorname{Ric}^{\sharp}\right)$ is already a multiplication operator with a real function and hence symmetric itself for all $\kappa$. For $\kappa=\frac{1}{2}$ we have:

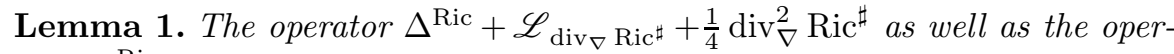
ator $\Delta^{\text {Ric }}+\mathscr{L}_{\operatorname{div}_{\nabla} \text { Ric }}$ are symmetric. 
We conclude this section with the computation of the covariant divergences of Ric ${ }^{\sharp}$ in the case of a Ricci type connection. In view of equation (3) and the fact that $\nabla_{X}$ commutes with $\sharp$, we have :

$$
\nabla_{X} \operatorname{Ric}^{\sharp}=\left(\nabla_{X} \text { Ric }\right)^{\sharp}=\frac{1}{2 n+1}\left(X^{b} \vee U^{b}\right)^{\sharp}=\frac{1}{2 n+1} X \vee \mathrm{U}
$$

whence

$$
\operatorname{div}_{\nabla} \operatorname{Ric}^{\sharp}=U .
$$

Moreover, from (4) and (5) we get

$$
\operatorname{div}_{\nabla}^{2}\left(\operatorname{Ric}^{\sharp}\right)=\operatorname{div}_{\nabla} \mathrm{U}=-\frac{2 n+1}{2(n+1)} K+2(n+1) f .
$$

We can also obtain from this the symmetric version of the Ricci operator, according to Lemma 1.

\section{The Weyl-Moyal Star Product}

After this excursion on the Ricci operator, we are now back to the situation of Section 1. On the big space $P$, the symplectic connection $\nabla$ is flat. We have thus a Weyl-Moyal star product on $P$, explicitly given by

$$
f \star g=\sum_{r=0}^{\infty} \frac{1}{r !}\left(\frac{\nu}{2}\right)^{r} C_{r}(f, g)
$$

for $f, g \in C^{\infty}(P)[[\nu]]$, where

$$
C_{r}(f, g)=\left\langle\Lambda_{P} \otimes \cdots \otimes \Lambda_{P}, \frac{1}{r !} \mathrm{D}^{r} f \otimes \frac{1}{r !} \mathrm{D}^{r} g\right\rangle
$$

and the natural pairing is done "over cross". Locally we have

$$
\begin{aligned}
C_{r}(f, g)= & \Lambda_{P}^{i_{1} j_{1}} \cdots \Lambda_{P}^{i_{r} j_{r}} \mathrm{i}_{\mathrm{s}}\left(\frac{\partial}{\partial x^{i_{1}}}\right) \cdots \mathrm{i}_{\mathrm{s}}\left(\frac{\partial}{\partial x^{i_{r}}}\right) \frac{1}{r !} \mathrm{D}^{r} f \\
& \times \mathrm{i}_{\mathrm{s}}\left(\frac{\partial}{\partial x^{j_{1}}}\right) \cdots \mathrm{i}_{\mathrm{s}}\left(\frac{\partial}{\partial x^{j_{r}}}\right) \frac{1}{r !} \mathrm{D}^{r} g .
\end{aligned}
$$

Since $\nabla$ is flat $\star$ defines an associative law on the space of formal power series in the parameter $\nu$ with coefficients in $C^{\infty}(P)$ [4]. The $C_{r}$ are bidifferential operators which are of order at most $r$ in each argument, and satisfy the symmetry condition $C_{r}(u, v)=(-1)^{r} C_{r}(v, u)$. These properties are summarized by saying that $\star$ is a natural star product of Weyl-type as.

We have now two derivations of $\star$ : first it follows directly from the fact that $\nabla$ is $S$-invariant and $\mathscr{L}_{S} \Lambda_{P}=-\Lambda_{P}$ that 


$$
\mathcal{E}=\nu \frac{\partial}{\partial \nu}+\mathscr{L}_{S}
$$

is a $\nu$-Euler derivation, i.e. a $\mathbb{C}$-linear derivation of $\star$, see e.g. [21]. Second, we consider the quasi-inner derivation $\frac{1}{\nu} \operatorname{ad}(H)$. Since $\mathrm{D}^{3} H=0$, only the terms of order $\nu^{1}$ and $\nu^{2}$ contribute. But thanks to the Weyl-type symmetry of $\star$, only odd powers of $\nu$ occur in commutators, this is immediate from (45). Thus we have

$$
\frac{1}{\nu} \operatorname{ad}(H) f=\frac{1}{\nu} \nu\{H, f\}=X_{H}(f),
$$

which shows that $X_{H}$ is a quasi-inner derivation. With other words, $\star$ is strongly invariant with respect to the (classical) momentum map $H$. This strong invariance will allow us to use the phase space reduction also for $\star$ to obtain a star product on $M$. To this end, we first note the following:

Lemma 2. There are unique bidifferential operators $\hat{C}_{r}^{\text {red }}$ on $M$ of order $r$ in each argument such that

$$
\operatorname{pr}^{*} u \star \operatorname{pr}^{*} v=\sum_{r=0}^{\infty} \frac{1}{r !}\left(\frac{\nu}{2}\right)^{r} \frac{1}{H^{r}} \operatorname{pr}^{*} \hat{C}_{r}^{\text {red }}(u, v)
$$

In particular, we have $\hat{C}_{1}^{\mathrm{red}}(u, v)=\left\langle\Lambda_{M}, \mathrm{~d} u \otimes \mathrm{d} v\right\rangle$.

Proof. Clearly, $H^{r} C_{r}\left(\mathrm{pr}^{*} u, \mathrm{pr}^{*} v\right)$ is invariant under $X_{H}$ and homogeneous of degree 0 under $S$, hence a pull-back of a function $\hat{C}_{r}^{\text {red }}(u, v) \in C^{\infty}(M)$ via pr. This defines $\hat{C}_{r}^{\text {red }}$ uniquely. The statement on the order of differentiation is straightforward. Finally, $\hat{C}_{1}^{\text {red }}$ is obtained from (17).

Remark 2. Though it seems tempting, the operators $\hat{C}_{r}^{\text {red }}$ do not combine into a star product on $M$ directly: the prefactors $H^{r}$ spoil the associativity as one can show by a direct computation. Hence we will need a slightly more involved reduction.

We will need the second order term of $H \star f$ with an arbitrary function $f \in C^{\infty}(P)$. By the symmetry properties of (45) we know that $C_{2}(H, f)=$ $C_{2}(f, H)$.

Proposition 1. Let $f \in C^{\infty}(P)$, then

$$
\begin{aligned}
C_{2}(f, H)=\frac{1}{H}( & -\frac{1}{n+1}\left(\Delta^{\mathrm{Ric}}\right)^{\text {hor }} f-\frac{1}{2} \mathscr{L}_{\text {Vhor }} f-\operatorname{pr}^{*}(\phi) \mathscr{L}_{S}^{2} f \\
& \left.+\left(\mathscr{L}_{\text {Vhor }} \mathscr{L}_{S}+\mathscr{L}_{S} \mathscr{L}_{\text {Vhor }}\right) f+\frac{1}{2} \mathscr{L}_{X_{H}}^{2} f\right)
\end{aligned}
$$

where $\left(\Delta^{\mathrm{Ric}}\right)^{\text {hor }} f$ is the pairing of the horizontal lift of the 2-tensor Ric $^{M \#}$ with the second covariant derivative with respect to the flat connection $\nabla$ on $P$. Precisely, in a local chart, it is given by 


$$
\operatorname{pr}^{*}\left(\operatorname{Ric}^{M \sharp i j}\right)\left[\mathscr{L}_{\partial_{i}^{\text {hor }}} \mathscr{L}_{\partial_{j}^{\text {hor }}} f-\operatorname{pr}^{*}\left(\Gamma_{i j}^{M k}\right) \mathscr{L}_{\partial_{k}^{\text {hor }}} f+\frac{1}{n+1} \operatorname{pr}^{*} \operatorname{Ric}_{i j}^{M} \mathscr{L}_{S} f\right] .
$$

Observe that $\operatorname{Ric}^{M \sharp i j} \operatorname{Ric}_{i j}^{M}=-\operatorname{tr}\left(\rho^{2}\right)$.

Proof. First we note that $\mathrm{i}_{\mathrm{s}}(X) \mathrm{D}^{2} f=2 \nabla_{X} \mathrm{~d} f$ by the very definition of $\mathrm{D}$. Thus using (16) we can compute $C_{2}(f, g)$ for $f, g \in C^{\infty}(P)$ explicitly and get

$$
\begin{aligned}
C_{2}(f, g) & =\frac{1}{H^{2}}\left(\operatorname{pr}^{*}\left(\Lambda_{\text {red }}^{i j} \Lambda_{\text {red }}^{k l}\right)\left(\nabla_{\partial_{k}^{\text {hor }}} \mathrm{d} f\right)\left(\partial_{i}^{\text {hor }}\right)\left(\nabla_{\partial_{l}^{\text {hor }}} \mathrm{d} g\right)\left(\partial_{j}^{\text {hor }}\right)\right. \\
& +\operatorname{pr}^{*}\left(\Lambda_{\text {red }}^{k l}\right)\left(\nabla_{\partial_{k}^{\text {hor }} \mathrm{d} f}\right)(S)\left(\nabla_{\partial_{l}^{\text {hor }} \mathrm{d} g}\right)\left(X_{H}\right) \\
& +\operatorname{pr}^{*}\left(\Lambda_{\text {red }}^{k l}\right)\left(\nabla_{\partial_{l}^{\text {hor }}} \mathrm{d} f\right)\left(X_{H}\right)\left(\nabla_{\partial_{k}^{\text {hor }}} \mathrm{d} g\right)(S) \\
& +\operatorname{pr}^{*}\left(\Lambda_{\text {red }}^{i j}\right)\left(\nabla_{S} \mathrm{~d} f\right)\left(\partial_{i}^{\text {hor }}\right)\left(\nabla_{X_{H}} \mathrm{~d} g\right)\left(\partial_{j}^{\text {hor }}\right) \\
& +\operatorname{pr}^{*}\left(\Lambda_{\text {red }}^{i j}\right)\left(\nabla_{X_{H}} \mathrm{~d} f\right)\left(\partial_{j}^{\text {hor }}\right)\left(\nabla_{S} \mathrm{~d} g\right)\left(\partial_{i}^{\text {hor }}\right) \\
& +\left(\nabla_{S} \mathrm{~d} f\right)(S)\left(\nabla_{X_{H}} \mathrm{~d} g\right)\left(X_{H}\right)+\left(\nabla_{X_{H}} \mathrm{~d} f\right)\left(X_{H}\right)\left(\nabla_{S} \mathrm{~d} g\right)(S) \\
& \left.-\left(\nabla_{S} \mathrm{~d} f\right)\left(X_{H}\right)\left(\nabla_{X_{H}} \mathrm{~d} g\right)(S)-\left(\nabla_{X_{H}} \mathrm{~d} f\right)(S)\left(\nabla_{S} \mathrm{~d} g\right)\left(X_{H}\right)\right)
\end{aligned}
$$

where we have used local coordinates on $M$ as well as the horizontal lift according to (12). Next, one computes the second covariant derivatives of $H$ explicitly. One finds

$$
\begin{gathered}
\left(\nabla_{X^{\text {hor }}} \mathrm{d} H\right)\left(Y^{\text {hor }}\right)=-\operatorname{pr}^{*}(t(X, Y)) H \\
\left(\nabla_{X^{\text {hor }}} \mathrm{d} H\right)\left(X_{H}\right)=\operatorname{pr}^{*}(\omega(X, \mathrm{~V})) H=\left(\nabla_{X_{H}} \mathrm{~d} H\right)\left(X^{\text {hor }}\right) \\
\left(\nabla_{X^{\text {hor }}} \mathrm{d} H\right)(S)=0=\left(\nabla_{S} \mathrm{~d} H\right)\left(X^{\text {hor }}\right) \\
\left(\nabla_{X_{H}} \mathrm{~d} H\right)(S)=0=\left(\nabla_{S} \mathrm{~d} H\right)\left(X_{H}\right), \\
\left(\nabla_{X_{H}} \mathrm{~d} H\right)\left(X_{H}\right)=-\operatorname{pr}^{*}(\phi) H \quad \text { and } \quad\left(\nabla_{S} \mathrm{~d} H\right)(S)=\frac{1}{2} H
\end{gathered}
$$

where we used $\mathrm{d} H\left(X_{H}\right)=0$ and $\mathrm{d} H(S)=H$ as well as $\mathrm{d} H\left(X^{\text {hor }}\right)=0$ together with the explicit formulas (18) - (23). Putting things together gives the result thanks to the local form (31) for $\Delta^{\mathrm{Ric}}$ and $\operatorname{Ric}^{i j}=\Lambda_{M}^{i k} \Lambda_{M}^{j l} \operatorname{Ric}_{k l}$.

Definition 2. For $f \in C^{\infty}(P)$ we define the second order differential operator $\Delta$ by

$$
\Delta f=C_{2}(f, H) .
$$

As we shall see in the next section, this operator will be crucial for the construction of the reduced star product. 


\section{Reduction of the Star Product}

We come now to the reduction of $\star$. Here we follow essentially the BRST / Koszul approach advocated in [8] which simplifies drastically thanks to the codimension one reduction. Codimension one reductions have also been discussed by Glößner [19,20] and Fedosov [15], while the general case of reduction for coisotropic constraint manifolds is discussed in $[6,7,13,14]$. We shall briefly recall those aspects of [8] which are needed here.

We first consider the classical situation: the classical Koszul operator $\partial$ : $C^{\infty}(P) \longrightarrow C^{\infty}(P)$ is defined by

$$
\partial f=f(H-1)
$$

Next we define the classical homotopy $h: C^{\infty}(P) \longrightarrow C^{\infty}(P)$ by

$$
h f= \begin{cases}\frac{1}{H-1}\left(f-\operatorname{pr}_{1}^{*} \iota^{*} f\right) & \text { on } P \backslash \iota(\Sigma) \\ \mathscr{L}_{S} f & \text { on } \iota(\Sigma) .\end{cases}
$$

An easy argument shows that $h f$ is actually smooth. Then we have the homotopy formula

$$
f=\partial h f+\operatorname{pr}_{1}^{*} \iota^{*} f
$$

for $f \in C^{\infty}(P)$ together with the properties

$$
\iota^{*} \partial=0 \text { and } h \mathrm{pr}_{1}^{*}=0
$$

Moreover, $\partial, h, \iota^{*}$, and $\operatorname{pr}_{1}^{*}$ are equivariant with respect to the action of $X_{H}$ on $P$ and the Reeb vector field on $\Sigma$, respectively. The classical vanishing ideal $\mathcal{J}_{\Sigma}$ of $\Sigma$ is given by

$$
\mathcal{J}_{\Sigma}=\operatorname{ker} \iota^{*}=\operatorname{im} \partial
$$

by (60), and turns out to be a Poisson subalgebra of $C^{\infty}(P)$ as $\Sigma$ is coisotropic. Moreover,

$$
\mathcal{B}_{\Sigma}=\left\{f \in C^{\infty}(P) \mid\left\{f, \mathcal{J}_{\Sigma}\right\} \subseteq \mathcal{J}_{\Sigma}\right\}
$$

is the largest Poisson subalgebra of $C^{\infty}(P)$ such that $\mathcal{J}_{\Sigma} \subseteq \mathcal{B}_{\Sigma}$ is a Poisson ideal. It is well-known and easy to see that $\mathcal{B}_{\Sigma}$ can also be characterized by

$$
\mathcal{B}_{\Sigma}=\left\{f \in C^{\infty}(P) \mid \iota^{*} f \in C^{\infty}(\Sigma)^{Z}=\pi^{*} C^{\infty}(M)\right\}
$$

from which it easily follows that the Poisson algebra $\mathcal{B}_{\Sigma} / \mathcal{J}_{\Sigma}$ is isomorphic to the Poisson algebra $C^{\infty}(M)$ via $\iota^{*}$ and $\pi^{*}$.

We shall now deform the above picture according to [8] where we only use the 'Koszul part' of the BRST complex. First we define the quantum Koszul operator $\boldsymbol{\partial}: C^{\infty}(P)[[\nu]] \longrightarrow C^{\infty}(P)[[\nu]]$ by

$$
\partial f=f \star(H-1) \text {. }
$$


We set

$$
\mathcal{J}_{\Sigma}=\operatorname{im} \boldsymbol{\partial}=\left\{f \in C^{\infty}(P)[[\nu]] \mid f=g \star(H-1)\right\},
$$

which is the left ideal generated by $H-1$ with respect to $\star$. Next we consider

$$
\mathcal{B}_{\Sigma}=\left\{f \in C^{\infty}(P)[[\nu]] \mid\left[f, \mathcal{J}_{\Sigma}\right]_{\star} \subseteq \mathcal{J}_{\Sigma}\right\},
$$

which is the largest subalgebra of $C^{\infty}(P)[[\nu]]$ such that $\mathcal{J}_{\Sigma} \subseteq \mathcal{B}_{\Sigma}$ is a twosided ideal, the so-called idealizer of $\mathcal{J}_{\Sigma}$. The following simple algebraic lemma is at the core of Bordemann's interpretation of the reduction procedure $[6,7]$ :

Lemma 3. Let $\mathcal{A}$ be a unital $\mathbb{k}$-algebra and $\mathcal{J} \subseteq \mathcal{A}$ a left ideal. Let $\mathcal{B} \subseteq \mathcal{A}$ be the idealizer of $\mathcal{J}$ and $\mathcal{A}_{\text {red }}=\mathcal{B} / \mathcal{J}$. Then the left $\mathcal{A}$-module $\mathcal{A} / \mathcal{J}$ becomes a right $\mathcal{A}_{\text {red }}$-module via $[b]:[a] \mapsto[a b]$ for $[b] \in \mathcal{A}_{\text {red }}$ and $[a] \in \mathcal{A} / \mathcal{J}$, such that

$$
\mathcal{A}_{\text {red }} \cong \operatorname{End}_{\mathcal{A}}(\mathcal{A} / \mathcal{J})^{\text {opp }} \text {. }
$$

This way $\mathcal{A} / \mathcal{J}$ becomes a $\left(\mathcal{A}, \mathcal{A}_{\text {red }}\right)$-bimodule.

In our situation, we want to show that $C^{\infty}(P)[[\nu]] / \mathcal{J}_{\Sigma}$ provides a deformation of $C^{\infty}(\Sigma)$ and $\mathcal{B}_{\Sigma} / \mathfrak{J}_{\Sigma}$ induces a star product $\star_{\text {red }}$ on $M$. This can be done in great generality, in our situation the arguments simplify thanks to the codimension one case.

We define the quantum homotopy

$$
\boldsymbol{h}=h(\mathrm{id}+(\boldsymbol{\partial}-\partial) h)^{-1}
$$

and the quantum restriction map

$$
\iota^{*}=\iota^{*}(\mathrm{id}+(\boldsymbol{\partial}-\partial) h)^{-1},
$$

which are clearly well-defined since $\boldsymbol{\partial}-\partial$ is at least of order $\lambda$ and thus id $+(\boldsymbol{\partial}-\partial) h$ is invertible by a geometric series. From (60) we immediately find

$$
f=\partial \boldsymbol{h} f+\operatorname{pr}_{1}^{*} \iota^{*} f
$$

for all $f \in C^{\infty}(P)[[\nu]]$. Moreover, we still have the relations

$$
\iota^{*} \operatorname{pr}_{1}^{*}=\mathrm{id}_{C^{\infty}(\Sigma)[[\nu]]} \quad \text { and } \quad h \operatorname{pr}_{1}^{*}=0
$$

as in the classical case. Finally, all maps are still equivariant with respect to the flow of $X_{H}$ and $Z$, respectively.

Proposition 2. The functions $C^{\infty}(\Sigma)[[\nu]]$ becomes a left $\star$-module via

$$
f \bullet \psi=\iota^{*}\left(f \star \operatorname{pr}_{1}^{*} \psi\right) .
$$

This module structure is isomorphic to the module structure of $C^{\infty}(P)[[\nu]] / \mathcal{J}_{\Sigma}$ via $\operatorname{pr}_{1}^{*}$ and $\iota^{*}$. 
Proof. Since im $\boldsymbol{\partial}=\operatorname{ker} \boldsymbol{\iota}^{*}$ by (71), the quantum restriction map $\iota^{*}$ induces a linear bijection $C^{\infty}(P)[[\nu]] / \mathcal{J}_{\Sigma} \longrightarrow C^{\infty}(\Sigma)[[\nu]]$ whose inverse is induced by $\operatorname{pr}_{1}^{*}$ by (72). Then (73) is just the pulled-back module structure.

From the strong invariance of the star product $\star$ we obtain the following characterization of $\mathcal{B}_{\Sigma}$ :

Lemma 4. $\mathcal{B}_{\Sigma}=\left\{f \in C^{\infty}(P)[[\nu]] \mid \iota^{*} f \in \pi^{*} C^{\infty}(M)[[\nu]]\right\}$.

Proof. Indeed, on one hand we have $Z \iota^{*} f=-\nu \iota^{*}$ ad $(H) f$ by equivariance of $\iota^{*}$. Since for $f \in \mathcal{B}_{\Sigma}$ we have $\operatorname{ad}(H) f \in \mathfrak{J}_{\Sigma}$, this gives $\iota^{*} f=0$ by (71). Conversely, $\iota^{*} f=0$ implies $\operatorname{ad}(H) f \in \mathfrak{J}_{\Sigma}$ and hence $(H-1) \star f \in \mathfrak{J}_{\Sigma}$ from which $\mathfrak{J}_{\Sigma} \star f \subseteq \mathfrak{J}_{\Sigma}$ follows. But this implies $f \in \mathcal{B}_{\Sigma}$.

Theorem 2. The quotient $\mathcal{B}_{\Sigma} / \mathcal{J}_{\Sigma}$ is $\mathbb{C}[[\nu]]$-linearly isomorphic to $C^{\infty}(M)[[\nu]]$ via

$$
C^{\infty}(M)[[\nu]] \ni u \mapsto\left[\operatorname{pr}^{*} u\right] \in \mathcal{B}_{\Sigma} / \mathcal{J}_{\Sigma}
$$

with inverse induced by

$$
\mathcal{B}_{\Sigma} / \mathcal{J}_{\Sigma} \ni[f] \mapsto \iota^{*} f \in \pi^{*} C^{\infty}(M)[[\nu]] .
$$

This induces a deformed product $\star_{\mathrm{red}}$ for $C^{\infty}(M)[[\nu]]$ via

$$
\pi^{*}\left(u \star_{\mathrm{red}} v\right)=\iota^{*}\left(\operatorname{pr}^{*} u \star \operatorname{pr}^{*} v\right),
$$

which turns out to be a differential star product quantizing $\{\cdot, \cdot\}_{M}$. Finally, the bimodule structure of $C^{\infty}(\Sigma)[[\nu]]$ according to Lemma 3 and Proposition 2 is given by

$$
\psi \bullet u=\iota^{*}\left(\operatorname{pr}_{1}^{*} \psi \star \operatorname{pr}^{*} u\right) .
$$

Proof. For $u \in C^{\infty}(M)[[\nu]]$ we clearly have $\iota^{*} \operatorname{pr}^{*} u=\iota^{*} \operatorname{pr}_{1}^{*} \pi^{*} u=\pi^{*} u \in$ $\pi^{*} C^{\infty}(M)[[\nu]]$ whence $\operatorname{pr}^{*} u \in \mathcal{B}_{\Sigma}$ by Lemma 4 and (74) is well-defined. Since $\mathfrak{J}_{\Sigma}=\operatorname{ker} \iota^{*}$, by Lemma 4 it follows that (75) is well-defined and injective. Clearly, (74) and (75) are mutually inverse by (72) whence $\star_{\text {red }}$ is an associative $\mathbb{C}[[\nu]]$-bilinear product for $C^{\infty}(M)[[\nu]]$. It can be shown $[8$, Lem. 27] that there exists a formal series $S=\mathrm{id}+\sum_{r=1}^{\infty} \nu^{r} S_{r}$ of differential operators $S_{r}$ on $P$ such that $\iota^{*}=\iota^{*} \circ S$, from which it easily follows that $\star_{\text {red }}$ is bidifferential. Finally, computing the first orders of

$$
u \star_{\text {red }} v=\sum_{r=0}^{\infty} \frac{1}{r !}\left(\frac{\nu}{2}\right)^{r} C_{r}^{\mathrm{red}}(u, v)
$$

explicitly gives $C_{0}^{\text {red }}(u, v)=u v$ and $C_{1}^{\text {red }}(u, v)=\{u, v\}_{M}$, whence $\star_{\text {red }}$ is a star product quantizing the correct Poisson bracket. The last statement is clear by construction.

Up to now we just followed the general reduction scheme from [8] which simplifies for the codimension one case, see also $[19,20]$. Let us now bring our more specific features into the game: 
Lemma 5. Let $f \in C^{\infty}(P)^{X_{H}}[[\nu]]$ be invariant. Then

$$
(\boldsymbol{\partial}-\partial) f=\frac{\nu^{2}}{8} \Delta f .
$$

The operator $\Delta$ is invariant.

Proof. By invariance of $f$ we only have the second order term in the right multiplication by $H-1$, which was computed in Proposition 1. The invariance of $\Delta$ follows from the strong invariance of $\star$ by

$$
\begin{aligned}
X_{H}(\Delta f) & =X_{H}\left(f \star H-\frac{\nu}{2} C_{1}(f, H)-f H\right) \\
& =\frac{1}{\nu} \operatorname{ad}(H)(f \star H)+\frac{\nu}{2} X_{H}\left(X_{H}(f)\right)-X_{H}(f) H \\
& =\frac{1}{\nu}(\operatorname{ad}(H)(f)) \star H-\frac{\nu}{2} C_{1}\left(X_{H}(f), H\right)-X_{H}(f) H \\
& =\Delta\left(X_{H}(f)\right) .
\end{aligned}
$$

Lemma 6. Let $f \in C^{\infty}(P)^{X_{H}}[[\nu]]$ be invariant. Then

$$
\iota^{*} f=\iota^{*}\left(\mathrm{id}+\frac{\nu^{2}}{8} \Delta h\right)^{-1} f .
$$

Proof. Since $h$ and $\Delta$ preserve invariance this follows by induction from the last lemma.

Proposition 3. Let $u, v \in C^{\infty}(M)[[\nu]]$. Then $u \star_{\text {red }} v$ is determined by

$$
\pi^{*}\left(u \star_{\text {red }} v\right)=\iota^{*}\left(\sum_{r=0}^{\infty} \nu^{r} \sum_{2 s+t=r} \frac{1}{(-8)^{s} 2^{t} t !}(\Delta h)^{s}\left(\frac{1}{H^{t}} \operatorname{pr}^{*} \hat{C}_{t}^{\mathrm{red}}(u, v)\right)\right) .
$$

Proof. This is a simple consequence of Lemma 6 together with Lemma 2.

From this formula we see that we have to proceed in two steps in order to compute the true bidifferential operators $C_{r}^{\text {red }}$ out of the operators $\hat{C}_{r}^{\text {red }}$ : first we have to control $\Delta$ and $h$ applied to functions of the form $\frac{1}{H^{t}} \operatorname{pr}^{*} u$. Second, the application of $\iota^{*}$ simply sets $H=1$ and gives $\iota^{*} \operatorname{pr}^{*}=\pi^{*}$, whence this part can be considered to be trivial.

Lemma 7. Let $u \in C^{\infty}(M)$ and $k \in \mathbb{N}$. Then

$$
h\left(\frac{1}{H^{k}} \operatorname{pr}^{*} u\right)=-\left(\frac{1}{H}+\cdots+\frac{1}{H^{k}}\right) \operatorname{pr}^{*} u .
$$

Proof. On $P \backslash \iota(\Sigma)$ we have from (59) 


$$
\begin{aligned}
h\left(\frac{1}{H^{k}} \operatorname{pr}^{*} u\right) & =\frac{1}{H-1}\left(\frac{1}{H^{k}} \operatorname{pr}^{*} u-\operatorname{pr}_{1}^{*} \iota^{*}\left(\frac{1}{H^{k}} \operatorname{pr}^{*} u\right)\right) \\
& =\frac{1}{H-1}\left(\frac{1}{H^{k}}-1\right) \operatorname{pr}^{*} u \\
& =-\left(\frac{1}{H}+\cdots+\frac{1}{H^{k}}\right) \operatorname{pr}^{*} u .
\end{aligned}
$$

By continuity this extends also to $\iota(\Sigma)$.

In particular, applying the homotopy $h$ to a linear combination of functions of the form $\frac{1}{H^{k}} \operatorname{pr}^{*} u$ gives again such a linear combination.

Lemma 8. Let $u \in C^{\infty}(M)$ and $k \in \mathbb{N}$. Then

$$
\begin{aligned}
\Delta\left(\frac{1}{H^{k}} \operatorname{pr}^{*} u\right)=- & \frac{1}{n+1} \frac{1}{H^{k+1}} \operatorname{pr}^{*}\left(\Delta^{\mathrm{Ric}} u\right. \\
& \left.+\frac{4 k+1}{2 n+1} \mathscr{L}_{\mathrm{U}} u+\frac{k}{n+1} \operatorname{tr}\left(\varrho^{2}\right) u+\frac{4 k^{2}}{2 n+1} \mathrm{f} u\right) .
\end{aligned}
$$

Proof. This follows from $\mathscr{L}_{X_{H}} H=0=\mathscr{L}_{X^{\text {hor }}} H$ and $\mathscr{L}_{S} H=H$ together with $\mathscr{L}_{X^{\text {hor }}} \operatorname{pr}^{*} u=\operatorname{pr}^{*} \mathscr{L}_{X} u$ and $\mathscr{L}_{X_{H}} \operatorname{pr}^{*} u=0=\mathscr{L}_{S} \operatorname{pr}^{*} u$ as well as Proposition 1.

Again, we see that applying $\Delta$ to this particular class of functions reproduces such linear combinations, though, of course, the combinatorics gets involved. In contrast to (82), the function $u$ is differentiated in (83).

Remark 3. From the above two lemmas the star product $\star_{\text {red }}$ can be computed by Proposition 3 in all orders. However, the explicit evaluation of the iteration $(\Delta h)^{s}$ seems to be tricky: the combinatorics gets quite involved, even in the case, where $\nabla^{M}$ is symmetric, i.e $\mathrm{U}=0$ and $\mathrm{f}=$ const, $\operatorname{tr}\left(\varrho^{2}\right)=$ const. In this particular case only the Ricci operator $\Delta^{\text {Ric }}$ has to be applied successively to the $\hat{C}_{r}^{\text {red }}$ to produce the $C_{r}^{\text {red }}$, including, of course, still some non-trivial combinatorics.

\section{Properties of the Reduced Star Product}

In this section we collect some further properties of the reduced star product.

Proposition 4. The star product $\star_{\text {red }}$ is of Weyl-type, i.e. the bidifferential operators $C_{r}^{\text {red }}$ satisfy

$$
\overline{C_{r}^{\mathrm{red}}(u, v)}=C_{r}^{\mathrm{red}}(\bar{u}, \bar{v})
$$

and

$$
C_{r}^{\mathrm{red}}(u, v)=(-1)^{r} C_{r}^{\mathrm{red}}(v, u) .
$$

In particular, complex conjugation becomes $a^{*}$-involution 


$$
\overline{u \star_{\text {red }} v}=\bar{v} \star_{\text {red }} \bar{u},
$$

where $\bar{\nu}=-\nu$ by definition. Moreover, $\star_{\text {red }}$ is natural in the sense of [22], i.e. $C_{r}^{\text {red }}$ is a bidifferential operator of order $r$ in each argument.

Proof. The operators $\hat{C}_{r}^{\text {red }}$ clearly satisfy (84) and (85). From (78) it follows that the operators $C_{r}^{\text {red }}$ are real operators, i.e. they satisfy (84), since $\Delta$ and

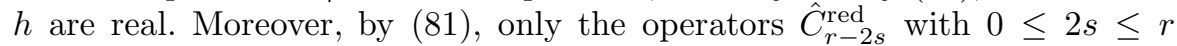
contribute to $C_{r}^{\text {red }}$ whence $C_{r}^{\text {red }}$ also satisfy (85) as $2 s$ is even. Then (86) follows. Moreover, $\hat{C}_{r}^{\text {red }}$ is a differential operator of order $r$ in each argument. Since the application of $\Delta h$ to $\frac{1}{H^{k}} \mathrm{pr}^{*} u$ by Lemma 7 and Lemma 8 gives a second order differential operator on the part $\operatorname{pr}^{*} u$, we conclude that $C_{r}^{\text {red }}$ is of order $r$ in each argument, too.

In a next step, we explicitly compute the second order term $C_{2}^{\text {red }}$ of $\star_{\text {red }}$. According to [22], the second order term of a natural star product determines uniquely a symplectic connection: in our case, we reproduce the Ricci type connection $\nabla^{M}$.

Proposition 5. Let $u, v \in C^{\infty}(M)[[\nu]]$. Then

$$
C_{2}^{\mathrm{red}}(u, v)=\left\langle\Lambda_{\text {red }} \otimes \Lambda_{\text {red }}, \frac{1}{2} \mathrm{D}_{M}^{2} u \otimes \frac{1}{2} \mathrm{D}_{M}^{2} v\right\rangle+\frac{2}{n+1}\left\langle\mathrm{Ric}^{\sharp}, \mathrm{d} u \otimes \mathrm{d} v\right\rangle .
$$

In particular, the symplectic connection determined by $\star_{\text {red }}$ is $\nabla^{M}$.

Proof. From (81) we see that

$$
\begin{aligned}
\pi^{*} C_{2}^{\mathrm{red}}(u, v) & =8 \iota^{*}\left(\sum_{2 s+t=2} \frac{1}{(-8)^{s} 2^{t} t !}(\Delta h)^{s}\left(\frac{1}{H^{t}} \operatorname{pr}^{*} \hat{C}_{2}^{\mathrm{red}}(u, v)\right)\right) \\
& =\iota^{*}\left(\frac{1}{H^{2}} \operatorname{pr}^{*} \hat{C}_{2}^{\mathrm{red}}(u, v)-\Delta h \operatorname{pr}^{*} \hat{C}_{0}^{\mathrm{red}}(u, v)\right) \\
& =\pi^{*} \hat{C}_{2}^{\mathrm{red}}(u, v)
\end{aligned}
$$

since $h \mathrm{pr}^{*}=0$ by the very definition (59) of $h$. Thus $C_{2}^{\text {red }}=\hat{C}_{2}^{\text {red }}$ in this order of $\nu$. The corrections to the terms $\hat{C}_{r}^{\text {red }}$ start only in order $r \geq 3$. To compute $\hat{C}_{2}^{\text {red }}$ we need the second covariant derivatives $\nabla \mathrm{dpr}^{*} u$ of pull-backs $\operatorname{pr}^{*} u$. Here we obtain

$$
\begin{gathered}
\left(\nabla_{X^{\text {hor }}} \operatorname{dpr}^{*} u\right)\left(Y^{\text {hor }}\right)=\operatorname{pr}^{*}\left(\left(\nabla_{X}^{M} \mathrm{~d} u\right)(Y)\right) \\
\left(\nabla_{X^{\text {hor }}} \operatorname{dpr}^{*} u\right)\left(X_{H}\right)=-\operatorname{pr}^{*}(\mathrm{~d} u(\tau X))=\left(\nabla_{X_{H}} \operatorname{dpr}^{*} u\right)\left(X^{\text {hor }}\right) \\
\left(\nabla_{X^{\text {hor }}} \operatorname{dpr}^{*} u\right)(S)=-\frac{1}{2} \operatorname{pr}^{*}(\mathrm{~d} u(X))=\left(\nabla_{S} \mathrm{dpr}^{*} u\right)\left(X^{\mathrm{hor}}\right) \\
\left(\nabla_{X_{H}} \mathrm{dpr}^{*} u\right)\left(X_{H}\right)=\operatorname{pr}^{*}(\mathrm{~d} u(\mathrm{~V}))
\end{gathered}
$$




$$
\left(\nabla_{X_{H}} \operatorname{dpr}^{*} u\right)(S)=\left(\nabla_{S} \operatorname{dpr}^{*} u\right)\left(X_{H}\right)=\left(\nabla_{S} \operatorname{dpr}^{*} u\right)(S)=0 .
$$

Inserting this into the general expression (51) for $C_{2}$ and using $\operatorname{pr}^{*} \hat{C}_{2}^{\text {red }}(u, v)=$ $H^{2} C_{2}\left(\mathrm{pr}^{*} u, \mathrm{pr}^{*} v\right)$ gives the result (87). From this, the last statement follows directly as the star product $\star_{\text {red }}$ is of Weyl type and the only second order terms in $C_{2}^{\text {red }}$ are described by using $\nabla^{M}$, see [22, Prop. 3.1].

\section{References}

1. Abraham, R., Marsden, J. E.: Foundations of Mechanics. Addison Wesley Publishing Company, Reading, Mass., 2. edition, 1985.

2. Baguis, P., Cahen, M.: A Construction of Symplectic Connections Through Reduction. Lett. Math. Phys. 57 (2001), 149-160.

3. Baguis, P., CAhen, M.: Marsden-Weinstein reduction for symplectic connections. Bull. Belg. Math. Soc. Simon Stevin 10.1 (2003), 91-100.

4. Bayen, F., Flato, M., Frønsdal, C., Lichnerowicz, A., Sternheimer, D.: Deformation Theory and Quantization. Ann. Phys. 111 (1978), 61-151.

5. Bieliavsky, P., Cahen, M., Gutt, S., Rawnsley, J., Schwachhöfer, L.: Symplectic connections. Int. J. Geom. Methods Mod. Phys. 3.3 (2006), 375-420.

6. Bordemann, M.: (Bi)Modules, morphismes et réduction des star-produits : le cas symplectique, feuilletages et obstructions. Preprint math.QA/0403334 (2004), 135 pages.

7. Bordemann, M.: (Bi)Modules, morphisms, and reduction of star-products: the symplectic case, foliations, and obstructions. Trav. Math. 16 (2005), 9-40.

8. Bordemann, M., Herbig, H.-C., Waldmann, S.: BRST Cohomology and Phase Space Reduction in Deformation Quantization. Commun. Math. Phys. 210 (2000), 107-144.

9. Bordemann, M., Neumaier, N., Pflaum, M. J., Waldmann, S.: On representations of star product algebras over cotangent spaces on Hermitian line bundles. J. Funct. Anal. 199 (2003), 1-47.

10. Bordemann, M., Neumaier, N., Waldmann, S.: Homogeneous Fedosov Star Products on Cotangent Bundles I: Weyl and Standard Ordering with Differential Operator Representation. Commun. Math. Phys. 198 (1998), 363-396.

11. Cahen, M., Gutt, S., Schwachhöfer, L.: Construction of Ricci-type connections by reduction and induction. In: MARsden, J. E., RATiu, T. S. (EDS.): The breadth of symplectic and Poisson geometry, vol. 232 in Progress in Mathematics, 41-57. Birkhäuser Boston, Boston, MA, 2005. Festschrift in honor of Alan Weinstein.

12. Cahen, M., Schwachhöfer, L. J.: Special symplectic connections and Poisson geometry. Lett. Math. Phys. 69 (2004), 115-137.

13. Cattaneo, A. S., Felder, G.: Coisotropic Submanifolds in Poisson Geometry and Branes in the Poisson Sigma Model. Lett. Math. Phys. 69 (2004), 157-175.

14. Cattaneo, A. S., Felder, G.: Relative formality theorem and quantisation of coisotropic submanifolds. Preprint math.QA/0501540 (2005), 31 pages.

15. Fedosov, B. V.: Reduction and Eigenstates in Deformation Quantization. In: Demuth, M., Schrohe, E., Schulze, B.-W. (EDs.): Pseudo-differential Calculus and Mathematical Physics, vol. 5 in Advances in Partial Differential Equations, 277-297. Akademie Verlag, Berlin, 1994. 
16. Fedosov, B. V.: Non-Abelian Reduction in Deformation Quantization. Lett. Math. Phys. 43 (1998), 137-154.

17. Fisch, J., Henneaux, M., Stasheff, J., Teitelboim, C.: Existence, uniqueness and cohomology of the classical BRST charge with ghosts of ghosts. Comm. Math. Phys. 120 (1989), 379-407.

18. Gerstenhaber, M.: On the Deformation of Rings and Algebras. Ann. Math. 79 (1964), 59-103.

19. GLÖssner, P.: Phasenraumreduktion von Sternprodukten für superauf lösbare Constraintalgebren. $\mathrm{PhD}$ thesis, Fakultät für Physik, Albert-LudwigsUniversität, Freiburg, 1998.

20. Glössner, P.: Star Product Reduction for Coisotropic Submanifolds of Codimension 1. Preprint Freiburg FR-THEP-98/10 math.QA/9805049 (May 1998).

21. Gutt, S., RAWNSLEY, J.: Equivalence of star products on a symplectic manifold; an introduction to Deligne's Čech cohomology classes. J. Geom. Phys. 29 (1999), 347-392.

22. Gutt, S., Rawnsley, J.: Natural Star Products on Symplectic Manifolds and Quantum Moment Maps. Lett. Math. Phys. 66 (2003), 123-139.

23. VAisman, I.: Symplectic curvature tensors. Monatsh. Math. 100.4 (1985), 299327. 